\title{
What Can Neuroscience Tell Us about the Hard Problem of Consciousness?
}

\author{
Berit Brogaard $^{1,2}$ and Dimitria Electra Gatzia ${ }^{3 *}$ \\ ${ }^{1}$ Brogaard Lab for Multisensory Research, University of Miami, Coral Gables, FL, USA, ${ }^{2}$ Department of Philosophy, \\ University of Oslo, Oslo, Norway, ${ }^{3}$ Department of Philosophy, University of Akron Wayne College, Orrville, OH, USA
}

Keywords: consciousness, paradigm, psychotropics, schizophrenia, metastability, neuroimaging

\section{OPEN ACCESS}

Edited by:

Chrystalina A. Antoniades,

University of Oxford, UK

Reviewed by:

Alexander Fingelkurts,

Brain and Mind Technologies

Research Centre (BM-Science),

Finland

Aldo Lucion,

Universidade Federal do Rio Grande do Sul, Brazil

Alessandro Capucci,

Marche Polytechnic University, Italy

Alain Morin

Mount Royal University, Canada

*Correspondence:

Dimitria Electra Gatzia dg29@uakron.edu

Specialty section:

This article was submitted to Neuroprosthetics,

a section of the journal

Frontiers in Neuroscience

Received: 01 February 2016

Accepted: 12 August 2016

Published: 07 September 2016

Citation:

Brogaard B and Gatzia DE (2016) What Can Neuroscience Tell Us about the Hard Problem of Consciousness?

Front. Neurosci. 10:395.

doi: 10.3389/fnins.2016.00395
A main research goal within neuroscience is to explain the relation between neurophysiological processes and conscious experiences. One approach involves focusing on problems such as the integration of information, the deliberate control of behavior, the ability to discriminate and categorize environmental stimuli, etc. These problems have been dubbed by philosophers as "easy" to suggest that the present limitations hindering progress could be overcome by more sophisticated methods in the near future (Chalmers, 1995, 1996). For example, explaining the integration of information requires describing the neurophysiological mechanisms responsible for information processing. Although these mechanisms are not currently well known, it is very likely that neuroscience will be able to explain them in the near future. It has been argued, however, that the problem of phenomenal consciousness cannot be explained by reference to such mechanisms because it involves a special kind of subjective qualities, i.e., phenomenal qualities that are present in experience (Dennett, 1991, 2003; Block, 1995; Chalmers, 1996; Revonsuo, 2006; Majeed, 2016). For example, the experience of seeing a red patch has a different phenomenal quality (a redly quality or "feel") from the experience of seeing a green patch (a greenly quality or "feel")1. The problem of explaining how or why neurophysiological processing gives rise to phenomenal experiences has been dubbed the "hard problem of consciousness" to suggest that solving it requires a paradigm shift in neuroscience (Chalmers, 1995, 1996).

There are several theoretical frameworks which attempt to integrate brain and mind (Crick and Koch, 1990, 2003; Edelman and Tononi, 2000; Revonsuo, 2000, 2003; Baars, 2003; Zeki, 2003, 2004; Freeman, 2007). However, most of them attempt to explain phenomenal consciousness through its neural correlates (Rees et al., 2002; Noë and Thompson, 2004). At the core of these theoretical frameworks is the idea that by isolating the physical processes associated with conscious experiences we can find comprehensive, systematic associations between them and characteristics of conscious experience. According to one such theory, phenomenal consciousness arises from a correspondence between the structural properties of the information processed in the brain and structural properties of conscious experience (see for example, Kohler, 1947). According to another such theory, consciousness arises from synchronized oscillations in the cerebral cortex (Crick and Koch, 1995). However, these theories have several drawbacks (Fingelkurts et al., 2009). Most notably, they rely on the relation of correlation, which is too weak to have any explanatory power (Chalmers, 1997; Noë and Thompson, 2004; Revonsuo, 2006; Fingelkurts et al., 2009) or postulate entities that cannot be easily measured scientifically (Revonsuo, 2000; Seth et al., 2008).

An alternative theoretical framework, known as Operational Architectonics, has recently been proposed to explain phenomenal consciousness (Fingelkurts et al., 2009, 2010). It does not reduce phenomenal experience to the brain functions since it is believed that such a reduction would either

\footnotetext{
${ }^{1}$ The presence of an external stimulus is not necessary for having a conscious experience with a certain phenomenal quality; hallucinating a pink elephant involves having a conscious experience with a certain phenomenal quality in the absence of a sensory stimulus.
} 
distort or eliminate consciousness. Rather, it purports to establish that the level of organization in the cortex is functionally isomorphic to the phenomenal level of experience. In other words, the phenomenological structure of consciousness corresponds to the brain's structure of operational architectonics (Fingelkurts and Fingelkurts, 2001; Fingelkurts et al., 2010). On this view, the cortical system can perform many operations, involving large-scale local and global cortical networks, characterized by different spatial and temporal parameters, using one or more operational modules. These operational modules are not directly connected with structural or static functional modules in the brain. Rather, they have a dynamic nature-they are metastable-and exist in their own operational space-time, i.e., an abstract space and time the brain constructs each time an operational module emerges (Fingelkurts and Fingelkurts, 2001; Fingelkurts et al., 2009, 2010) ${ }^{2}$. These metastable brain states underlie complex brain functions and corresponding conscious complex operations such as cognitive percepts and mental states that are representational in nature (Fingelkurts and Fingelkurts, 2001).

Rapid advances in the field of neuroimaging techniques including magnetoencephalography (MEG), electroencephalography (EEG), functional MRI (fMRI), diffusion tensor imaging (DTI), voxel based morphomentry (VBM), and optical imaging, have allowed neuroscientists to investigate neurophysiological processes in ways that have not been possible until recently (Tehovnik et al., 2006; Bandettini, 2009). Combining these techniques with advanced analysis procedures during different conditions such as hypnosis, psychiatric and neurological conditions, subliminal stimulation, and psychotropic drugs (Bhagwagar et al., 2006; Griffiths et al., 2008; Studerus et al., 2011; Lee and Roth, 2012) began transforming the study of neuroscience in ways that it is now possible to test such frameworks, bringing us a step closer to solving the hard problem of consciousness.

A study using a task-free functional MRI (fMRI) protocol designed to capture the transition from normal waking consciousness to the psychedelic state and blood-oxygen leveldependent (BOLD) fMRI to map cerebral blood flow and changes in venous oxygenation before and after intravenous infusions of placebo and psilocybin found that psilocybin significantly decreases the positive coupling of medial prefrontal cortex and posterior cingulate cortex (two key structural hubs) (Carhart-Harris et al., 2012; see also Lee and Roth, 2012) ${ }^{3}$. Although, the function of the posterior cingulate cortex is not yet fully understood, its association with the default-mode network regions (Raichle et al., 2001), known to host the

\footnotetext{
${ }^{2}$ Metastability initiates the hierarchical coupling between phenomenal and neurophysiological states while simultaneously allowing them to retain their individuality (Fingelkurts et al., 2009). It expresses the full complexity of a system, accommodates heterogeneous elements, does not require disengagement mechanisms in order for a system to switch to another state, and allows a system to flexibly survey through a set of possibilities (see Kelso and Tognoli, 2007).

${ }^{3}$ For studies using varying neuroimaging techniques such as arterial spin labeling (ASL), blood oxygen level-dependent (BOLD) measures, magnetoencephalography (MEG), or electroencephalography (EEG) and varying psychotropic substances such as LSD and ayahuasca (see Riba et al., 2002; Stuckey et al., 2005; Carhart-Harris et al., 2016).
}

highest number of cortico-cortical connections in the brain, suggests that it may play a role in conscious experience (Raichle, 1998; Gusnard et al., 2001) and may account for findings indicating that functional decreases in activity in the posterior cingulate cortex alter the conscious states of subjects under the influence of psilocybin (Carhart-Harris et al., 2012; Wood et al., 2012).

Neuroimaging techniques also indicate that psychotropic drugs can modify the natural metastable organization of brain activity (Fingelkurts et al., 2009). For example, a study in which healthy subjects were administered lorazepam (a benzodiazepine) showed that the number and strength of functional cortical connections increased significantly. These cortical changes were found to correspond to mental changes such as slowing of thinking and cognition (Fingelkurts et al., 2004). Such findings are consistent with the Operational Architecture framework, which posits that changes at mental level must be accompanied by corresponding changes in the metastable organization of brain activity (Fingelkurts et al., 2009).

A number of studies using neuroimaging techniques have also begun to examine the neural correlates of the phenomenal characteristics associated with auditory hallucinations in schizophrenic patients. Reduction in the prefrontal functional connectivity was found in studies using functional magnetic resonance imaging (fMRI) on eight schizophrenia patients and 10 controls in schizophrenia suffering from auditory hallucinations (Lawrie et al., 2002; see also Jardri et al., 2011; Allen et al., 2012). Although, more studies are needed, schizophrenia can provide an alternative approach to studying the functional connectivity of the brain and its relation to the resulting altered phenomenal states. For example, it is suggested that the altered phenomenal states in schizophrenia may be the result of a disruption of the metastable balance between large-scale integration and independent processing in the cortex, leading to an excess of local information expression by cortical areas (Bressler, 2003; Fingelkurts et al., 2009). Such suggestions could be tested in future studies.

The recent paradigm shift in neuroscience, which involves testing competing theoretical frameworks using a combination of neuroimaging techniques and advanced analysis procedures during different conditions, may allow us to find an adequate solution to the hard problem of consciousness. For example, the Operational Architecture framework posits that every change in the mental level must be accompanied by a corresponding change at the neurophysiological level (Fingelkurts et al., 2009). Unlike other frameworks, which rely on the relation of correlation that is too weak to have any explanatory power, this framework provides scientifically plausible ways to investigate the hard problem of consciousness. Different conditions such as psychotropic drugs, hypnosis, and schizophrenia can be used to test the hypothesis that mental changes must be accompanied by corresponding changes at the neurophysiological level. At the same time, neuroimaging techniques such as fMRI or EEG can be used to test whether changes at the neurophysiological level involve metastable changes between different functional operational modules in the cortex. It should nevertheless be emphasized that research on the hard problem of consciousness is currently in its 
infancy as a result of its perplexing nature. Although, the current paradigm shift discussed here may better prepare researchers to tackle the hard problem of consciousness, a considerable amount of research is required in order to arrive at any firm conclusions.

\section{REFERENCES}

Allen, P., Modinos, G., Hubl, D., Shields, G., Cachia, A., Jardri, R., et al. (2012). Neuroimaging auditory hallucinations in schizophrenia: from neuroanatomy to neurochemistry and beyond. Schizophr. Bull. 38, 695-703. doi: $10.1093 /$ schbul/sbs066

Bandettini, P. A. (2009). What's new in neuroimaging methods? Ann. N.Y. Acad. Sci. 1156, 260-293. doi: 10.1111/j.1749-6632.2009.04420.x

Baars, B. J. (2003). "How does a serial, integrated, and very limited stream of consciousness emerge from a nervous system that is mostly unconscious, distributed, parallel, and of enormous capacity?" in Essential Sources in the Scientific Study of Consciousness, eds B. J. Baars, W. P. Banks, and J. B. Newman (Cambridge, MA: MIT Press), 1123-1129.

Bhagwagar, Z., Hinz, R., Taylor, M., Fancy, S., Cowen, P., and Grasby, P. (2006). Increased 5-HT(2A) receptor binding in euthymic, medication-free patients recovered from depression: a positron emission study with [(11) C] MDL 100,907. Am. J. Psychiatry 163, 1580-1587. doi: 10.1176/ajp.2006.163.9.1580

Block, N. (1995). On the confusion about a function of consciousness. Behav. Brain Sci. 18, 227-288.

Bressler, S. (2003). Cortical coordination dynamics and the disorganization syndrome in schizophrenia. Neuropsychopharmacology 28(Suppl. 1), S35-S39. doi: $10.1038 /$ sj.npp.1300145

Carhart-Harris, R. L., Erritzoe, D., Williams, T., Stone, J. M., Reed, L. J., Colasanti, A., et al. (2012). Neural correlates of the psychedelic state as determined by fMRI studies with psilocybin. Proc. Natl. Acad. Sci. U.S.A. 109, 2138-2143. doi: 10.1073/pnas.1119598109

Carhart-Harris, R. L., Muthukumaraswamy, S., Roseman, L., Kaelen, M., Droog, W., Murphy, K., et al. (2016). Neural correlates of the LSD experience revealed by multimodal neuroimaging. Proc. Natl. Acad. Sci. U.S.A. 113, 4853-4858. doi: 10.1073/pnas.1518377113

Chalmers, D. J. (1995). Facing up to the problem of consciousness. J. Consc. Stud. 2, 200-219.

Chalmers, D. J. (1996). The Conscious Mind. New York, NY: Oxford University Press.

Chalmers, D. J. (1997). Moving forward on the problem of consciousness. J. Consc. Stud. 4, 3-46.

Crick, F., and Koch, C. (1995). Why neuroscience may be able to explain consciousness. Sci. Am. 273, 84-85.

Crick, F., and Koch, C. (1990). Towards a neurobiological theory of consciousness. Semin. Neurosci. 2, 263-275.

Crick, F., and Koch, C. (2003). A framework for consciousness. Nat. Neurosci. 6, 119-126. doi: 10.1038/nn0203-119

Dennett, D. C. (1991). Consciousness Explained. New York, NY: Little, Brown and Company.

Dennett, D. C. (2003). Explaining the "magic" of consciousness. J. Cultur. Evol. Psychol. 11, 7-19. doi: 10.1556/JCEP.1.2003.1.2

Edelman, G. M., and Tononi, G. (2000). A Universe of Consciousness: How Matter Becomes Imagination. New York, NY: Basic Books.

Fingelkurts, A. A., and Fingelkurts, A. A. (2001). Operational architectonics of the human brain biopotential field: towards solving the mind-brain problem. Brain Mind 2, 261-296. doi: 10.1023/A:1014427822738

Fingelkurts, A. A., Fingelkurts, A. A., Kivisaari, R., Pekkonen, E., Ilmoniemi, R. J., and Kähkönen, S. A. (2004). Local and remote functional connectivity of neocortex under the inhibition influence. Neuroimage 22, 1390-1406. doi: 10.1016/j.neuroimage.2004.03.013

Fingelkurts, A. A., Fingelkurts, A. A., and Neves, C. F. H. (2009). Phenomenological architecture of a mind and operational architectonics of the brain: the unified metastable continuum. New Math. Nat. Comput. 5, 221-244. doi: 10.1142/S1793005709001258

\section{AUTHOR CONTRIBUTIONS}

All authors listed, have made an equal, substantial, direct and intellectual contribution to the work, their names appear alphabetically, and approved it for publication.

Fingelkurts, A. A., Fingelkurts, A. A., and Neves, C. F. H. (2010). Natural world physical, brain operational, and mind phenomenal space-time. Phys. Life Rev. 7, 195-249. doi: 10.1016/j.plrev.2010.04.001

Freeman, W. J. (2007). Indirect biological measures of consciousness from field studies of brains as dynamical systems. Neural Netw. 20, 1021-1031. doi: 10.1016/j.neunet.2007.09.004

Griffiths, R. R., Richards, W. A., McCann, U. D., and Jesse, R. (2008). Mysticaltype experiences occasioned by psilocybin mediate the attribution of personal meaning and spiritual significance 14 months later. J. Psychopharmacol. 22, 621-632. doi: 10.1177/0269881108094300

Gusnard, D. A., Akbudak, E., Shulman, G. L., and Raichle, M. E. (2001). Medial prefrontal cortex and self-referential mental activity: relation to a default mode of brain function. Proc. Natl. Acad. Sci. U.S.A. 98, 4259-4264. doi: 10.1073/pnas. 071043098

Jardri, R., Pouchet, A., Pins, D., and Thomas, P. (2011). Cortical activations during auditory verbal hallucinations in schizophrenia: a coordinate-based metaanalysis. Am. J. Psychiatry 168, 73-81. doi: 10.1176/appi.ajp.2010.09101522

Kelso, J. A. S., and Tognoli, E. (2007). "Toward a complementary neuroscience: metastable coordination dynamics of the brain," in Neurodynamics of Cognition and Consciousness, eds L. I. Perlovsky and R. Kozma (Heidelberg: Springer), 39-59.

Kohler, D. (1947). Gestalt Psychology. New York, NY: Liveright Publishing Corporation.

Lawrie, S. M., Buechel, C., Whalley, H. C., Frith, C. D., Friston, K. J., and Johnstone, E. C. (2002). Reduced frontotemporal functional connectivity in schizophrenia associated with auditory hallucinations. Biol. Psychiatry 51, 1008-1011.

Lee, H. M., and Roth, B. L. (2012). Hallucinogen actions on human brain revealed. Proc. Natl. Acad. Sci. U.S.A. 109, 1820-1821. doi: 10.1073/pnas.1121358109

Majeed, R. (2016). The hard problem and its explanatory targets. Ratio 29, 298-311. doi: $10.1111 /$ rati.12103

Noë, A., and Thompson, E. (2004). Are there neural correlates of consciousness? J. Consc. Stud. 11, 3-28.

Raichle, M. E. (1998). The neural correlates of consciousness: An analysis of cognitive skill learning. Philos. Trans. R. Soc. Lond. B Biol. Sci. 353, 1889-1901.

Raichle, M. E., MacLeod, A. M., Snyder, A. Z., Powers, W. J., Gusnard, D. A., and Shulman, G. L. (2001). A default mode of brain function. Proc. Natl. Acad. Sci. U.S.A. 98, 676-682. doi: 10.1073/pnas.98.2.676

Rees, G., Kreiman, G., and Koch, C. (2002). Neural correlates of consciousness in humans. Nat. Rev. Neurosci. 3, 261-270. doi: 10.1038/nrn783

Revonsuo, A. (2000). "Prospects for a scientific research program on consciousness," in Neural Correlates of Consciousness: Empirical and Conceptual Questions, ed T. Metzinger (Cambridge, MA: MIT Press), 57-75.

Revonsuo, A. (2003). The contents of phenomenal consciousness: one relation to rule them all and in the unity bind them. Psyche 9 . Available online at: http:// psyche.cs.monash.edu.au/v9/psyche-9-08-revonsuo.html

Revonsuo, A. (2006). Inner Presence: Consciousness as a Biological Phenomenon. Cambridge, MA: MIT Press.

Riba, J., Anderer, P., Morte, A., Urbano, G., Jané, F., Saletu, B., et al. (2002). Topographic pharmaco-EEG mapping of the effects of the South American psychoactive beverage ayahuasca in healthy volunteers. Br. J. Clin. Pharmacol. 53, 613-628. doi: 10.1046/j.1365-2125.2002.01609

Seth, A. K., Dienes, Z., Cleeremans, A., Overgaar, M., and Pessoa, L. (2008). Measuring consciousness: relating behavioural and neurophysiological approaches. Trends Cogn. Sci. 12, 314-321. doi: 10.1016/j.tics.2008.04.008

Stuckey, D. E., Lawson, R., and Luna, L. E. (2005). EEG gamma coherence and other correlates of subjective reports during ayahuasca experiences. $J$. Psychoactive Drugs 37, 163-178. doi: 10.1080/02791072.2005.10399798

Studerus, E., Kometer, M., Hasler, F., and Vollenweider, F. X. (2011). Acute, subacute and long-term subjective effects of psilocybin in healthy humans: a 
pooled analysis of experimental studies. J. Psychopharmacol. 25, 1434-1452. doi: $10.1177 / 0269881110382466$

Tehovnik, E. J., Tolias, A. S., Sultan, F., Slocum, W. M., and Logothetis, N. K. (2006). Direct and indirect activation of cortical neurons by electrical microstimulation. J. Neurophysiol. 96, 512-521. doi: 10.1152/jn. 00126.2006

Wood, J., Kim, Y., and Moghaddam, B. (2012). Disruption of prefrontal cortex large scale neuronal activity by different classes of psychotomimetic drugs. J. Neurosci. 32, 3022-3031. doi: 10.1523/JNEUROSCI.6377-11.2012

Zeki, S. (2003). The disunity of consciousness. Trends Cogn. Sci. 7, 214-218. doi: 10.1016/S1364-6613(03)00081-0

Zeki, S. (2004). "Insights into visual consciousness," in Human Brain Function, eds R. S. J. Frackowiak, K. J. Friston, C. D. Frith, R. J. Dolan, C. J.
Price, S. Zeki, J. Ashburner, and W. Penny (San Diego, CA: Academic Press).

Conflict of Interest Statement: The authors declare that the research was conducted in the absence of any commercial or financial relationships that could be construed as a potential conflict of interest.

Copyright $\odot 2016$ Brogaard and Gatzia. This is an open-access article distributed under the terms of the Creative Commons Attribution License (CC BY). The use, distribution or reproduction in other forums is permitted, provided the original author(s) or licensor are credited and that the original publication in this journal is cited, in accordance with accepted academic practice. No use, distribution or reproduction is permitted which does not comply with these terms. 